\title{
Bus Based Mobile Virus Diagnostic Laboratory for COVID-19 Sample Collection and Testing in Remote Areas
}

\author{
Y Sreenivas $\mathrm{Rao}^{1, *}$ and S Rajeshwar Rao \\ ${ }^{1}$ PGAD, Research Centre Imarat, Telangana, India \\ ${ }^{2}$ Defence Research and Development Organization, Ministry of Defense, Government of India, Telangana, India
}

${ }^{*}$ Corresponding author: Y Sreenivas Rao, Outstanding Scientist, PGAD, Research Centre Imarat, Hyderabad, Telangana, India, E-mail: ysr@rcilab.in

Received: 20 Jul, 2020 | Accepted: 31 Jul, 2020 | Published: 07 Aug, 2020

Citation: Rao YS, Rao SR (2020) Bus Based Mobile Virus Diagnostic Laboratory for COVID-19 Sample Collection and Testing in Remote Areas. J Emerg Dis Virol 6(1): dx.doi.org/10.16966/2473-1846.155

Copyright: (c) 2020 Rao YS, et al. This is an open-access article distributed under the terms of the Creative Commons Attribution License, which permits unrestricted use, distribution, and reproduction in any medium, provided the original author and source are credited.

\begin{abstract}
World Health Organization (WHO) declared Public Health Emergency of International Concern on 30 January, 2020 and named the outbreak caused by SARS-CoV-2 virus as COVID-19. This disease is spreading globally and it is very important to take necessary measures to contain it. The rapidly spreading, highly contagious and pathogenic Severe Acute Respiratory Syndrome-Coronavirus-2 (SARS-CoV-2) is transmitted human-to-human, has continuously spread to over 200 countries. It is imperative to immediately build, expand, train and deploy health workers to find, test, isolate and treat every case and trace every contact.

It would take a long time, a large staff and many resources to construct a new fixed bio-safety facility, thus delaying the prevention and control of the pandemic. Therefore, a mobile laboratory with both bio-safety and flexibility urgently needed to manage pandemic and emergent public health incidents.

To meet this requirement, authors have designed a Bus Based Mobile Virus Diagnostic Laboratory. This laboratory can be built on an existing chassis of a bus; it will have all the features and equipment required for sample collection, sample processing, sample storage and waste disposal. As it is on a mobile platform, it can reach any epicenter of the pandemic and public health authorities can initiate the screening process at a faster pace.

As per WHO and DBT/ICMR guidelines, a BSL-2 laboratory should be provided with negative pressure; airlock and HEPA filter-based air supply and exhaust system. In this paper, the design of BSL-2 laboratory built on a bus chassis platform of $12 \mathrm{~m}$ long ad $2.6 \mathrm{~m}$ wide, meeting above guidelines and that can function as a standalone Testing Center for COVID-19 is discussed.
\end{abstract}

Keywords: COVID-19; Mobile laboratory; Bio-Safety; Containment

\section{Introduction}

During the ongoing COVID-19 situation, to contain the spread of the disease, testing is a very important aspect. However, it will take a lot of effort and time to build fixed bio-safety laboratories for testing.

To meet this requirement, authors have designed a Bus Based Mobile Virus Diagnostic Laboratory. This laboratory can be built on an existing chassis of a bus; it will have all the features and equipment required for sample collection, sample processing, and sample storage, testing and waste disposal. As it is on a mobile platform, it can reach any epicenter of the pandemic and public health authorities can initiate the screening process at a faster pace.

In the past, there have been a number of models of mobile virology laboratories deployed in response to various outbreaks for diagnosis, surveillance, clinical management, research and military purposes. These mobile laboratories are either based on a container platform providing limited mobility or on truck platform providing mobility into the territories of the outbreak. Upon a literature survey, the following details of mobile laboratories deployed for various requirements in different countries can be found.

During the outbreak of Ebola, China deployed a truck-based Mobile BSL-3 laboratory in Sierra Leone to support the diagnosis of EBOV. For this purpose, three trucks mounted with standard shipping containers, comprising of main laboratory containers, auxiliary containers, and command container are employed. Laboratory equipment included qPCR and Bio Safety Cabinets. The laboratories were powered by alternating two $200 \mathrm{~kW}$ diesel generators [1].

Russia also deployed a truck-based mobile laboratory to Guinea under their Rospotrebnadzor's (Federal Service for Surveillance on Consumer Rights and Human Wellbeing) Specialized Antiepidemic 
Unit (SAEU). Mc Namara, et al. [2]; Russian Ministry of Finance, 2014; UNOG, 2016, 2017).

In response to the Marburg outbreak in 2005, WHO deployed the Mobile Laboratory Unit in support of patient management and epidemiological surveillance in the epicenter of the outbreak, Uige, Angola [3]. This Mobile Laboratory Unit (MLU) operated over 88 days and tested 620 oral swab/EDTA blood specimens from 388 individuals. The MLU operation allowed a diagnostic response in less than 4 hours from sample receiving and emerged as an essential part of an outbreak investigation.

A mobile DNA laboratory developed by The Forensic Science Institute of French "Gendarmerie Nationale" to perform genetic analysis in the context of crime scenes, terrorism attacks and disasters is used to support the hospital task force in Paris during the peak of the COVID-19 epidemic. Touron P, et al. have successfully adapted this autonomous Mobile DNA Laboratory to assist in COVID-19 diagnostic [4].

In response to the COVID-19 pandemic in India, Rao YS has developed a Mobile Virology Research and Diagnostic Laboratory (MVRDL) which is an autonomous, transportable BSL-2 \& 3 laboratory-based inside two shipping containers. MVRDL has RTPCR, ELISA reader, Nucleic acid extraction system, BSC and is in use for COVID-19 diagnosis [5].

Parsons A, et al. [6] have discussed in detail, utility and readiness of mobile and field transportable laboratories for bio-defence and global health security-related purposes.

In the current work, authors have designed a BSL-2 laboratory based on a bus platform, further adding to the mobility of the laboratory. This laboratory is provided with airlock and change rooms. The main BSL-2 area is provided with 20pa negative pressure, HEPA filter based supply-exhaust system, generator-based power supply and all the equipment that are required for a fully functional standalone laboratory for sample collection, testing for COVID-19. Detailed design philosophy and protocols for entry/exit of personnel and samples is explained below.

\section{Design Philosophy of Mobile BSL-2 Lab}

A bio-safety laboratory design is carried out by following referenced guidelines and enabling required features for:

- Smooth operation as per Laboratory bio-safety containment policy

- Specimen collection, inactivation and storage

- Specimen Testing

- Storage of Lab supplies and PPE kits

- Liquid and solid waste disposal

- De-contamination of the laboratory

\section{Design}

It is proposed to build a BSL-2 laboratory on a mobile chassis of 12 meters length. Fully built bus has dimensions of 12 meters length, 2.58 meters width and 3.8 meters height. This laboratory is fitted with diesel generator based power supply.

\section{Schematics of the laboratory (Figures 1-3)}

BSL-2 lab area: As per WHO and DBT/ICMR guidelines [7,8], BSL-2 laboratory must be equipped with a Bio-safety cabinet, Freezers to store samples, eyewash/sink, for disposal of other waste produced in the lab. Autoclave for disposal of bio-waste and Dunk tank is optional.

In this laboratory, the BSL-2 area is designed following relevant guidelines. This lab is equipped with Bio safety cabinet of class-II A2, glove box with dynamic pass box for sample collection, freezers than can store samples at $-20^{\circ} \mathrm{C}$, and $4^{\circ} \mathrm{C}$, dunk tank, SS sink for eyewash and double door autoclave for waste disposal.

Bio-Safety Cabinet (BSC) is placed as much far away from the entry door as possible so that there is no disturbance to laminar airflow inside BSC. Other lab equipment as necessary may be placed in the lab equipment area as shown above.

Entry into the lab should be with proper approved PPE and should be controlled through access control. Man limit of 5 should be imposed in the main lab area.

The lab should be provided with a lighting system and CCTV surveillance system.

Laboratory should have joint less vinyl flooring with aluminium covings. The ceiling of the lab also should be joint less. The internal building finishes shall be monolithic, impervious, non-particle shredding, chemical resistant especially to Hypochlorite cleaning and suitable to withstand chemical use during decontamination/ fumigation.

All doors of the lab should be self lockable and access controlled.

Water supply to the lab is through 3 tanks placed above the bus body.

Fire safety alarm should be provided and proper fire tender equipment should be placed inside of the laboratory.

HVAC system: HVAC system should be designed with $70 \%$ recirculation through HEPA filters and 30\% exhaust through HEPA filters in the BSL-2 area. Air conditioning should be maintained in such a way that temperature inside the lab is $24 \pm 4^{\circ} \mathrm{C}$. Indicators for temperature and relative humidity should be indicated outside of the lab near to entryway. Inlet and Exhaust air system should be designed to make more than 12 air changes per hour.

Generator room, large equipment area, and control panels: This area of the laboratory is equipped with inlet and exhaust air handling units, HVAC System, power supply panel and lab control panels that control laboratory. Double door autoclave opens into this room thereby enabling cleaning and disposal of waste through this room. This area opens to outside through hinged double door for easy access. Negative pressure is not maintained in this area. Generator having the capacity to run all the equipment is also accommodated in this laboratory.

PPE donning room: In this area street clothes are removed by personnel and are stored and personnel shall wear approved PPE. At the time of exit, the same clothes shall be worn. Doors of the room are interlocked in such a way that both doors of this room cannot be opened once. Also, there has to be an indicator indicating someone is occupying this room.

PPE doffing room: PPE will be doffed in this room at the time of exit. Also, there will be provisions to store additional supplies of PPE in this area. This room also acts as an airlock. Doors of the room are interlocked in such a way that both doors of this room cannot be opened once. Also, there has to be an indicator indicating someone is occupying this room.

Maintenance of pressure differential: Pressure differential between various rooms should be maintained in such a way that air always flows 

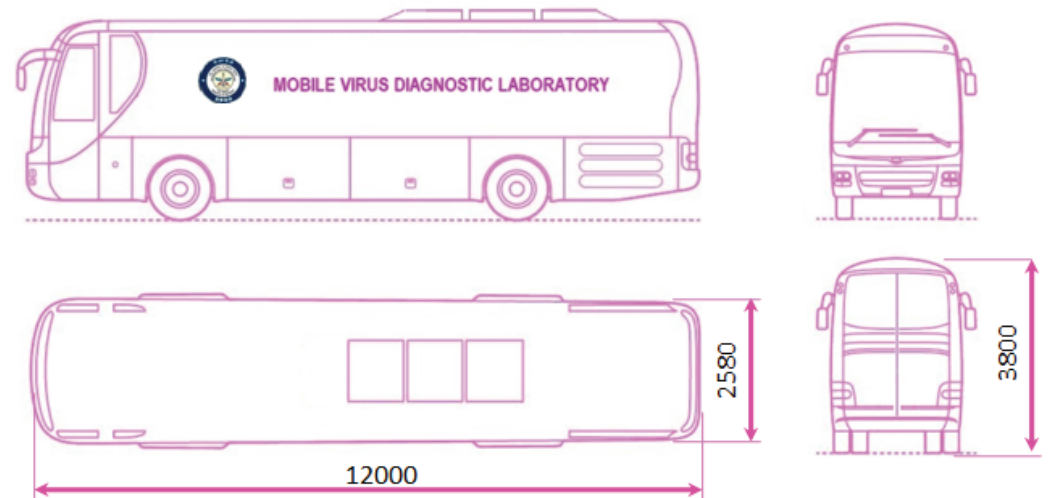

Figure 1: Schematics of the laboratory.
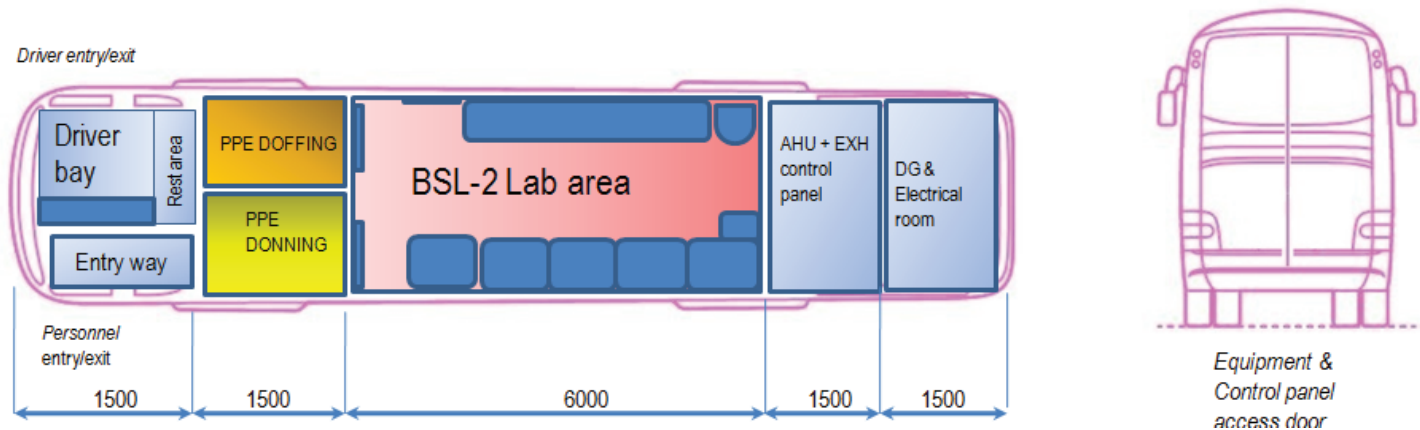

Figure 2: Layout details of sub-areas of the lab.

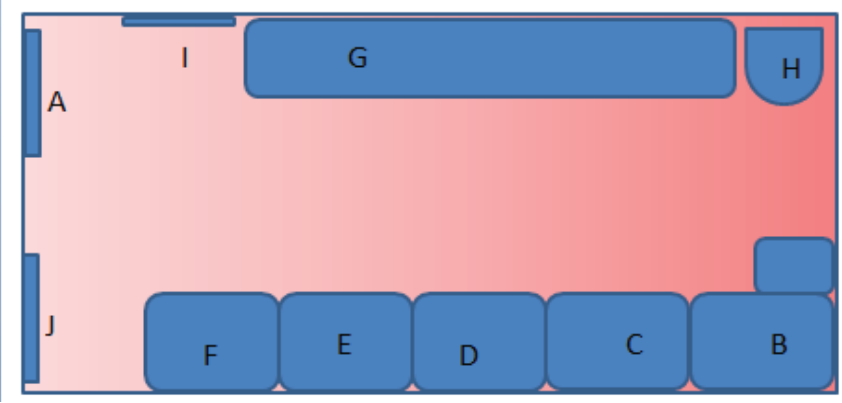
A - Entry
B - Sample Collection area
C - Bio safety cabinet
D \& E - Freezers
F- Lab equipment
G - Working table
$\mathrm{H}$ - Eye wash/sink
I-Emergency breakable window
J-Exit

Figure 3: Internal Layout details BSL-2 area.

from less contaminated areas to more contaminated areas. In Donning room pressure should be $-5 \mathrm{~Pa}$, in Doffing room pressure should be -10 $\mathrm{Pa}$ and inside BSL-2 area pressure should be $-20 \mathrm{~Pa}$ (Figure 4).

\section{Deployment of the laboratory}

Initially, wherever this laboratory is to be deployed, certain provisions have to be made. Suitable area, if possible isolated from habitation of size $50 \mathrm{mtr}$ X 50mtr should be chosen. The incineration pit is to be made as per guidelines for disposal of waste. Supply channel for a sample to be prepared. Suitable accommodation for personnel to be made and their entry channel to be established. A schematic for these provisions is shown in figure 5 .

\section{Sample collection method}

At sample collection location on the bus body, there will be a door that can be opened from outside when needed, as shown in the figure. Suspected patients will enter through the Sample receiving channel and will stand on a raised platform near this sample collection area of the bus. Nasopharyngeal/oropharyngeal swab of the patient is collected by a health care provider sitting inside of the bus through the glove box seeing through a viewing window. The sample will enter into the BSL-2 area of the laboratory through the dynamic pass box (Figure 6). 


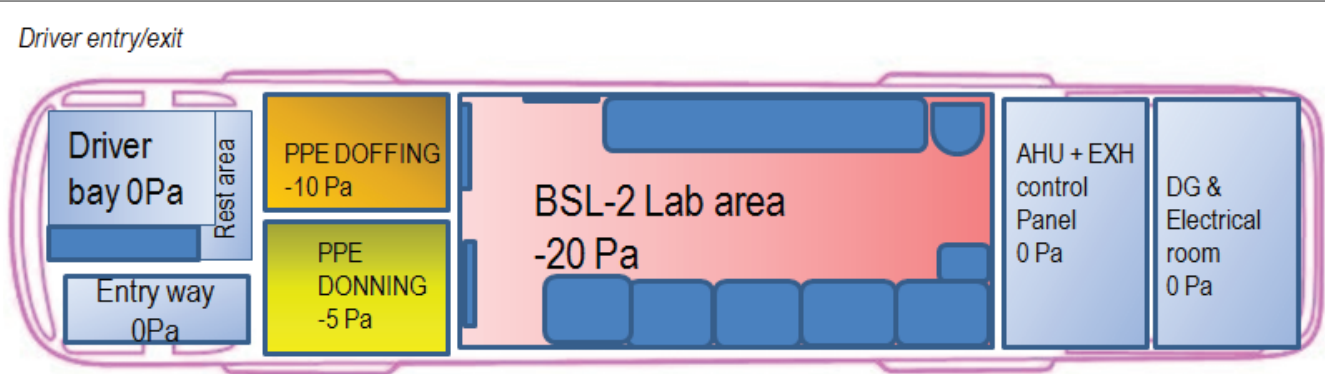

Personnel

entry/exit

Figure 4: Pressure differential scheme.

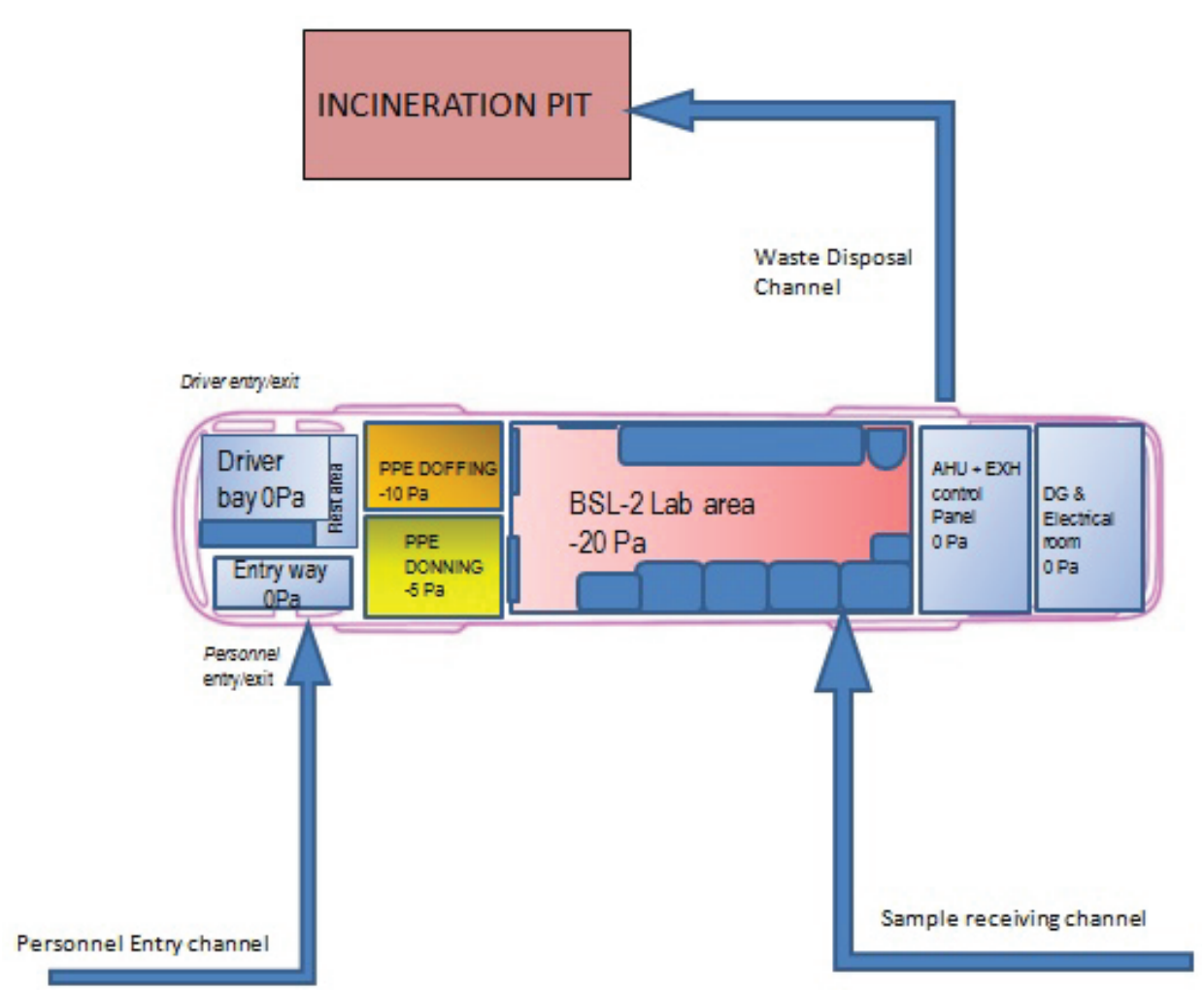

Figure 5: A schematic for Deployment of the laboratory.

\section{Entry/Exit protocols (Figure 7)}

Personnel entry: Personnel entry is through the main bus entry. Entry into the laboratory s through the access control system. Through stairs and entryway, personnel shall enter PPE Donning room where they will don PPE. From this room directly they enter the main lab area.

Personnel exit: For entry and exit there are separate doors.

1) Exit door or main lab opens into Doffing room where the personnel DOFF PPE and exit through PPE Donning room.

2) Finally, they wear street clothes in DONNING Room and exit the laboratory.
Material entry: All the samples are collected through the glove box and are transferred into the BSL-2 area through a dynamic pass box.

Waste disposal: Wastes are inactivated as per approved procedure; waste has to be disposed of in an incineration pit.

\section{Testing Equipments}

The bus should be equipped with all the testing equipment is required for sample collection, identification marking, storage, testing and waste disposal. The following types of equipment should be installed so those above functions of the laboratory are met. 


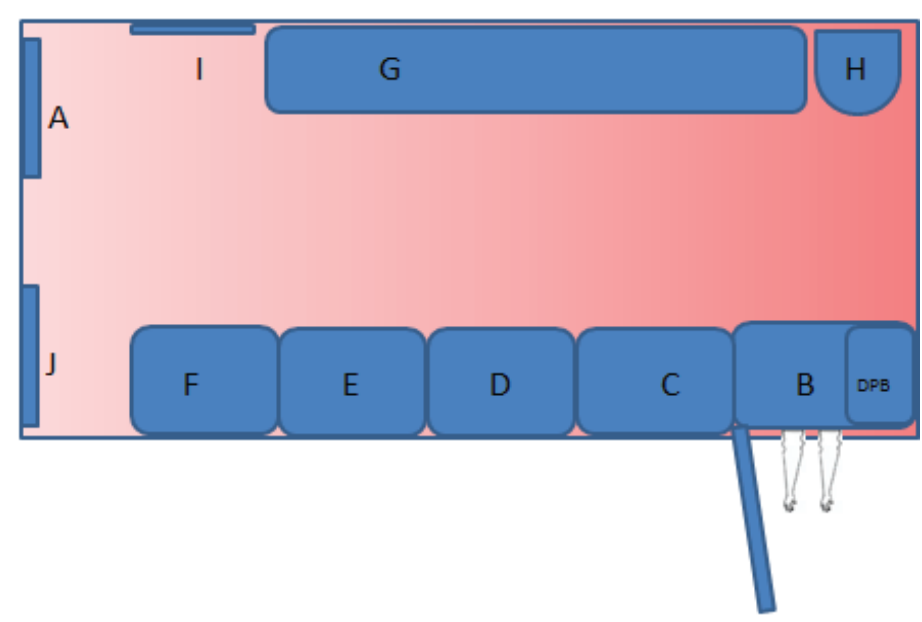

Figure 6: Sample collection method.

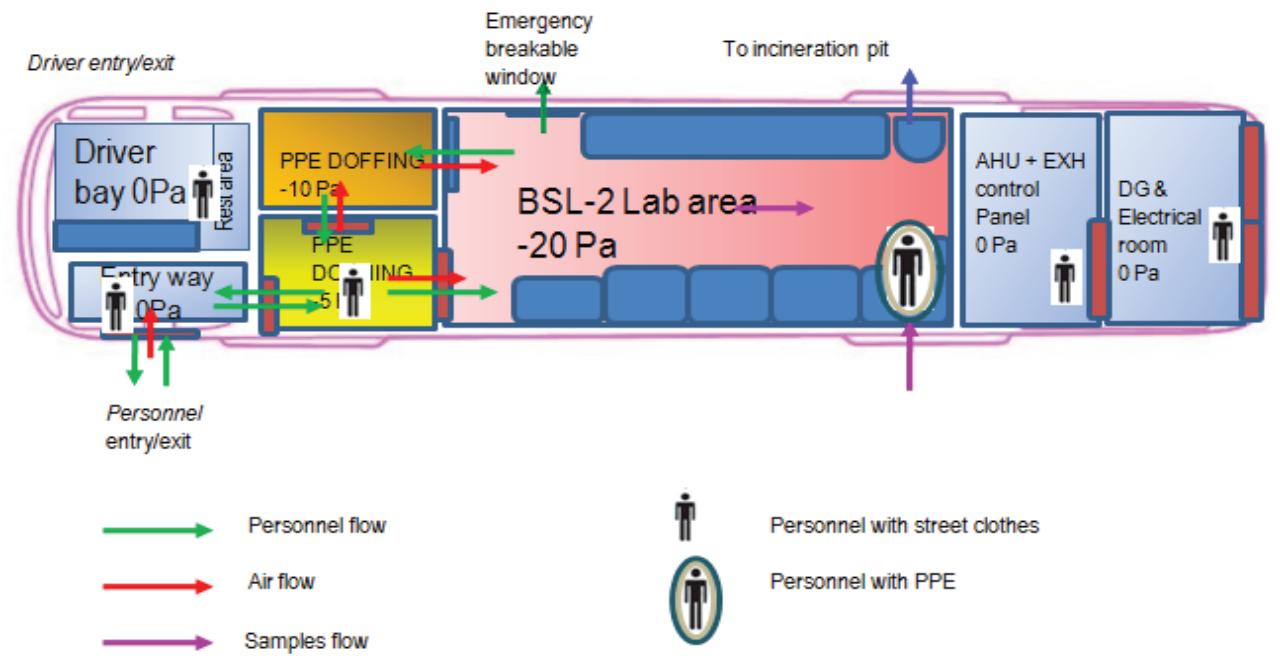

Figure 7: Entry/Exit protocols.

\begin{tabular}{|c|l|l|}
\hline S.No & \multicolumn{1}{|c|}{ Description } & \multicolumn{1}{|c|}{ Qty } \\
\hline 1 & Refrigerator, $4^{\circ} \mathrm{C}, 300$ lit capacity & 1 No \\
\hline 2 & Refrigerator, $-20^{\circ} \mathrm{C}, 340$ lit capacity & 1 No \\
\hline 3 & $\begin{array}{l}\text { Refrigerated centrifuge, } 0.2 \mathrm{ml} \text { to } 15 \mathrm{ml} \text { tubes capacity } \\
\text { rotor }\end{array}$ & 1 No \\
\hline 4 & ELISA reader with filter 0 to 4 OD & 1 No \\
\hline 5 & Real-time PCR Machine & 1 No \\
\hline 6 & Nucleic acid extraction system & 1 No \\
\hline 7 & Laptop with printer & 1 No \\
\hline 8 & Bar code maker with printer & 1 No \\
\hline 9 & Dunk Tank & 1 No \\
\hline 10 & $\begin{array}{l}\text { Micro pipetting device, 20, 100, 200 and } 1000 \text { micro } \\
\text { litre capacity }\end{array}$ & $\begin{array}{l}\text { Set } \\
4 \text { Nos }\end{array}$ \\
\hline 11 & Needle shredder & 1 No \\
\hline & & \\
\hline
\end{tabular}

\begin{tabular}{|l|l|l|}
\hline 12 & Bio-Safety Cabinet Class-II Type A2 & 1 No \\
\hline 13 & Double door Autoclave & 1 No \\
\hline
\end{tabular}

\section{Discussion and Conclusion}

In the current pandemic situation created by COVID-19, it is very essential to rapidly build and deploy facilities for sample collection and testing. However, in this situation, it is very difficult and timeconsuming to build fixed bio-safety laboratories for this purpose. Hence, authors have come up with a mobile solution called as Bus Based Mobile Virus Diagnostic Laboratory, which meets BSL-2 biosafety guidelines. Being mobile, such laboratories can be rapidly built and deployed in remotely located epicentres of the epidemic by various governments across the world. After the epidemic is over, this mobile laboratory can be used for research and training purposes. 


\section{References}

1. Zhang Y, Gong Y, Wang C, Liu W, Wang Z, et al. (2017) Rapid deployment of a mobile biosafety level-3 laboratory in Sierra Leone during the 2014 Ebola virus epidemic. PLoS Negl Trop Dis 11: e0005622.

2. Mc Namara T, Platonov A, Elleman T, Gresham L (2013) The humananimal inter-face and zoonotic threats: The Russian Federation approach. Biosecur Bioterror 11: 185-195.

3. Grolla A, Jones SM, Fernando L, Strong JE, Stroher U, et al. (2011) The use of a mobile laboratory unit in support of patient management and epidemiological surveillance during the 2005 Marburg Outbreak in Angola. PLoS Negl Trop Dis 5: e1183.

4. Touron P, Siatka C, Pussiau A, Follot S, Fritz T, et al. (2020) A mobile DNA laboratory for forensic science adapted to coronavirus SARSCoV-2 diagnosis. Eur J Clin Microbiol Infect Dis 1-4.
5. Rao YS (2020) Mobile Virology Research and Diagnostic Laboratory (MVRDL: BSL-3) for COVID-19 Screening, Virus Culturing and Vaccine Development. Trans Indian Natl Acad Eng 1-5.

6. Parsons A, Matero P, Adams M, Yeh K (2018) Examining the utility and readiness of mobile and field transportable laboratories for biodefence and global health security-related purposes. Global Security: Health, Science and Policy 3: 1-13.

7. WHO (2004) Laboratory Bio-safety Manual. Third Edition, World Health Organization, Geneva, Switzerland.

8. Department of Biotechnology, Ministry of Science and Technology, Government of India (2017) Regulations and Guidelines on Biosafety of Recombinant DNA Research \& Biocontainment. India. 